\title{
Effect of Pitch Type, Pitch Count, and Pitching Mechanics on Risk of Elbow and Shoulder Pain in Youth Baseball Pitchers
}

\author{
Stephen Lyman, ${ }^{*} \mathrm{PhD}$, Glenn S. Fleisig, ${ }^{*} \dagger \mathrm{PhD}$, James R. Andrews, ${ }^{*} \mathrm{MD}$, and \\ E. David Osinski,‡ MA
}

\begin{abstract}
From the *American Sports Medicine Institute and the $¥$ American Baseball Foundation, Birmingham, Alabama
\end{abstract}

\begin{abstract}
Background: Joint pain is thought to be an early sign of injury to a pitcher.
Objective: To evaluate the association between pitch counts, pitch types, and pitching mechanics and shoulder and elbow pain in young pitchers.

Study Design: Prospective cohort study.

Methods: Four hundred and seventy-six young (ages 9 to 14 years) baseball pitchers were followed for one season. Data were collected from pre- and postseason questionnaires, injury and performance interviews after each game, pitch count logs, and video analysis of pitching mechanics. Generalized estimating equations and logistic regression analysis were used.

Results: Half of the subjects experienced elbow or shoulder pain during the season. The curveball was associated with a $52 \%$ increased risk of shoulder pain and the slider was associated with an $86 \%$ increased risk of elbow pain. There was a significant association between the number of pitches thrown in a game and during the season and the rate of elbow pain and shoulder pain.

Conclusions: Pitchers in this age group should be cautioned about throwing breaking pitches (curveballs and sliders) because of the increased risk of elbow and shoulder pain. Limitations on pitches thrown in a game and in a season can also reduce the risk of pain. Further evaluation of pain and pitching mechanics is necessary.
\end{abstract}

๔ 2002 American Orthopaedic Society for Sports Medicine

Youth baseball pitchers are at risk for elbow and shoulder problems; however, the factors associated with these problems are poorly understood and have been infrequently studied. ${ }^{1,11,13,16,17}$ The risk of injury is known to increase with age and level of competition. ${ }^{15}$ It is believed that many of the pitching injuries that receive medical attention at higher competition levels result from cumulative microtrauma that began at the youth level. ${ }^{2}$

A recent study of youth baseball pitchers found that elbow pain was associated with age, weight, height, number of pitches thrown during a season, satisfaction with performance, fatigue, lifting weights, and playing outside of the league. ${ }^{14}$ Shoulder pain was associated with pitches thrown in a season, pitches thrown in a game, satisfaction,

† Address correspondence and reprint requests to Glenn S. Fleisig, PhD, American Sports Medicine Institute, 1313 13th Street South, Birmingham, AL 35205.

No author or related institution has received any financial benefit from research in this study. See "Acknowledgments" for funding information. and fatigue. Although that study showed associations between joint pain and risk factors, including number of pitches thrown, it did not show a statistically significant relationship between throwing breaking pitches and pain. Furthermore, the influence of pitching mechanics was not investigated.

The USA Baseball Medical \& Safety Advisory Committee recognized the need to improve the understanding of the factors associated with these injuries and commissioned the current study. Our two primary purposes were to improve the understanding of why youth baseball pitchers develop arm problems and to provide information for establishing safety regulations or recommendations.

\section{MATERIALS AND METHODS}

A prospective cohort study of players from 15 youth baseball parks throughout Alabama was conducted during the 1999 spring season. Park affiliations included Little League, Dixie Youth, and the Birmingham Metro League. 
A total of 147 coaches agreed to help implement this study. These coaches identified 539 potential pitchers for enrollment, of which 476 (88\%) pitched and agreed to participate. All participating coaches and pitchers were contacted to complete a baseline telephone questionnaire before the start of the season. This preseason telephone survey was used to collect demographic and baseball-related information as well as to identify pitchers with a previous history of arm problems.

Two hundred and forty of the potential pitchers volunteered to be videotaped before the start of the season. Of these $240,172(72 \%)$ pitched during the season and were eligible for study inclusion. These pitcher videos were qualitatively analyzed for proper pitching mechanics by using a model developed for this study by the American Sports Medicine Institute (ASMI) and the American Baseball Foundation (ABF). Twenty of these pitchers were also studied at the ASMI Biomechanics Laboratory to validate the ASMI/ABF qualitative model against laboratory data quantified with an automated digitizing system. This quantitative analysis has been shown to accurately assess pitching mechanics. ${ }^{7}$ With the qualitative model, 24 parameters of a pitcher's mechanics were rated as proper, insufficient, or excessive. Included were five parameters during windup and stride: initial movement, balance when lead knee is at maximum height, timing of hand separation, front leg swing, and arm path. Seven parameters at the instant the pitcher's front foot contacted the ground were rated: stride length, front knee flexion, front foot placement, front foot angle, arm position, shoulder abduction, and elbow flexion. Timing of trunk rotation, trunk arching, glove position, maximum elbow flexion, and maximum shoulder external rotation were assessed during delivery. Six parameters at the instant of ball release were rated: front knee flexion, lateral trunk tilt, forward trunk tilt, shoulder abduction, shoulder horizontal adduction, and elbow flexion. Trunk motion during follow-through was also assessed.

During the season, each team kept a pitch count log of game pitches thrown by each pitcher. There was no record of pitches thrown in nongame situations. The pitchers were interviewed after every game pitched by telephone by a university-based survey research unit. The interview questionnaire was similar to one that was shown by Lyman et al. ${ }^{14}$ to produce good inter- and intrarater reliability and that the young population largely understood. Questions asked included "Did your arm get tired while pitching?" "Did your arm get stiff or tight after pitching?" "Did you feel any pain in your elbow from pitching?" "Did you feel any pain in your shoulder from pitching?" and "Did you feel any pain in any other part of your arm from pitching?" With this series of questions, elbow pain and shoulder pain were distinguished from other complaints and were the only answers used in the statistical analysis. At the end of the season, all 476 pitchers were telephoned for a follow-up interview to identify factors that had the potential for change between the baseline interview and the end of the season. Included were questions about which types of pitches were thrown in games during the season.
Statistical analysis consisted of descriptive and inferential analyses. Descriptive statistics included the complaint rate (that is, the fraction of telephone interviews in which the pitcher reported joint pain) and the period prevalence (that is, the fraction of pitchers who had joint pain at least once during the study). Generalized estimating equations, a type of repeated measures analysis, were used to calculate game-specific risk and to adjust for changes in the dependent and independent variables over time. Logistic regression analysis was used to calculate overall risk and adjust for other independent variables. $P$ values were considered statistically significant if they were less than 0.05 .

The outcomes of interest were postgame complaints of elbow or shoulder pain. Injuries requiring medical treatment are rare for this age group, ${ }^{15}$ and this criterion was therefore not used as a measure of outcome. The primary independent variables of interest (and their sources) were types of pitches thrown (baseline/follow-up questionnaire), pitch counts (pitch count book), and pitching mechanics (video analysis). Pitch types of interest included the fastball, change-up, curveball, and slider. Pitch counts were calculated per game and per season.

\section{RESULTS}

The average age of study subjects was 12 years, with a fairly even age distribution among 9 - to 10 -year-old pitchers $(N=171), 11$ - to 12 -year-old pitchers $(N=154)$, and 13 - to 14-year-old pitchers $(N=151)$. The pitchers were an average height of 60 inches (1.52 meters) and had an average weight of 106 pounds (48 kg). There was one female pitcher in the study population. Nine percent of all subjects (43 of 476) were left-handed throwers.

The 476 pitchers made 3789 pitching appearances during the season. Nearly $7 \%$ of all pitching appearances $(254$ of 3789 ) resulted in elbow pain, with $28 \%$ of all subjects (134 of 476) reporting elbow pain at least once during the season (Table 1). More than $9 \%$ of all pitching appearances (353 of 3789 ) resulted in shoulder pain, with $35 \%$ of all subjects (165 of 476) reporting shoulder pain at least once during the season. Overall, almost $15 \%$ of all pitching appearances resulted in elbow or shoulder pain, with half $(50 \%)$ of all subjects reporting elbow or shoulder pain during the season.

Pitch types were evaluated for their relationship to both elbow and shoulder pain (Table 2). More than half of all pitchers threw a change-up and more than half threw a curveball. Use of the slider was much less common (14\%). Overall, only the slider was found to have a significant relationship to elbow pain, and this relationship was especially strong among 13- to 14-year-old pitchers (odds ratio of 3.49). There was an overall $86 \%$ increased risk of elbow pain among slider users. Use of the curveball accounted for a $52 \%$ increased risk of shoulder pain, without variation by age group. Use of the change-up pitch was associated with a $12 \%$ reduction in the risk of elbow pain and a $29 \%$ reduction in the risk of shoulder pain, although neither association was statistically significant.

The total number of pitches thrown in each appearance varied greatly, from 1 to 161 . For every age group studied, 
TABLE 1

Frequency of Joint Pain by Age and Associated Risk Factor

\begin{tabular}{|c|c|c|c|c|c|}
\hline \multirow{2}{*}{ Pain location and age } & \multirow{2}{*}{$\begin{array}{l}\text { No. of } \\
\text { pitchers }\end{array}$} & \multicolumn{2}{|c|}{ Appearances with pain } & \multicolumn{2}{|c|}{ Period prevalence } \\
\hline & & $N$ & $(\%)$ & $N$ & (\% of pitchers) \\
\hline \multicolumn{6}{|l|}{ Elbow } \\
\hline Overall & 476 & 254 & (6.7) & 134 & $(28)$ \\
\hline 9 to 10 years old & 171 & 65 & (4.7) & 37 & (22) \\
\hline 11 to 12 years old & 154 & 101 & (8.3) & 47 & (31) \\
\hline 13 to 14 years old & 151 & 88 & (7.4) & 50 & (33) \\
\hline \multicolumn{6}{|l|}{ Shoulder } \\
\hline Overall & 476 & 353 & $(9.3)$ & 165 & (35) \\
\hline 9 to 10 years old & 171 & 148 & (10.7) & 60 & (35) \\
\hline 11 to 12 years old & 154 & 81 & $(6.7)$ & 47 & (31) \\
\hline 13 to 14 years old & 151 & 124 & (10.4) & 58 & (38) \\
\hline \multicolumn{6}{|l|}{ Either location } \\
\hline Overall & 476 & 551 & $(14.6)$ & 237 & $(50)$ \\
\hline 9 to 10 years old & 171 & 187 & (13.5) & 78 & (46) \\
\hline 11 to 12 years old & 154 & 171 & (14.1) & 73 & (47) \\
\hline 13 to 14 years old & 151 & 193 & (16.2) & 86 & (57) \\
\hline
\end{tabular}

TABLE 2

Relationship Between Pitch Types and Arm Pain

\begin{tabular}{|c|c|c|c|c|c|}
\hline \multirow{2}{*}{ Pitch type and age } & \multirow{2}{*}{$\begin{array}{l}\text { No. of } \\
\text { pitchers }\end{array}$} & \multicolumn{2}{|c|}{ Elbow } & \multicolumn{2}{|c|}{ Shoulder } \\
\hline & & Odds ratio ${ }^{a}$ & $P$ value & Odds ratio $^{a}$ & $P$ value \\
\hline \multicolumn{6}{|l|}{ Change-up } \\
\hline Overall & 288 & 0.88 & 0.55 & 0.71 & 0.10 \\
\hline 9 to 10 years old & 69 & 1.41 & 0.36 & 0.65 & 0.20 \\
\hline 11 to 12 years old & 98 & 0.59 & 0.14 & 0.67 & 0.26 \\
\hline 13 to 14 years old & 121 & 0.69 & 0.37 & 1.10 & 0.83 \\
\hline \multicolumn{6}{|l|}{ Curveball } \\
\hline Overall & 252 & 1.14 & 0.54 & 1.52 & 0.04 \\
\hline 9 to 10 years old & 51 & 1.35 & 0.44 & 1.62 & 0.16 \\
\hline 11 to 12 years old & 77 & 1.06 & 0.86 & 1.54 & 0.22 \\
\hline 13 to 14 years old & 121 & 0.73 & 0.49 & 1.47 & 0.41 \\
\hline \multicolumn{6}{|l|}{ Slider } \\
\hline Overall & 65 & 1.86 & 0.03 & 1.77 & 0.38 \\
\hline 9 to 10 years old & 21 & 1.57 & 0.39 & 1.14 & 0.79 \\
\hline 11 to 12 years old & 16 & 0.74 & 0.61 & 3.38 & 0.02 \\
\hline 13 to 14 years old & 28 & 3.49 & $<0.01$ & 1.80 & 0.17 \\
\hline
\end{tabular}

${ }^{a}$ Referent group is fastball-only pitchers.

in $81 \%$ of the appearances (3075 of 3789 ) pitchers threw fewer than 75 pitches in at least $80 \%$ of the appearances. Overall, pitchers threw 100 or more pitches in a game in only $6 \%$ of the appearances (238 of 3789) (Table 3). Elbow pain was associated with an increasing number of game pitches, but the association was not quite statistically significant. In age-specific analysis, this trend was most apparent in the 13- to 14-year-old age group. There was a significant relationship between an increased number of game pitches and the risk of shoulder pain. When analyzed using age-specific data, this trend was significant in the 9- to 10-year-old and 13- to 14-year-old age groups.

Pitchers averaged 370 game pitches over the course of the season. There was a statistically significant relationship between pitches thrown in a season up through a given appearance and risk of elbow pain from that appearance (Table 4). A statistically significant relationship with a steadily increasing trend was also demonstrated for shoulder pain. Age-specific analysis (data not shown) did not reveal any appreciable differences.
Pitching mechanics evaluated with the ASMI/ABF qualitative model were weakly correlated with the mechanics quantified by the automatic digitizing system. Furthermore, none of the 24 pitching mechanics parameters analyzed with the qualitative model correlated with an increased risk of elbow or shoulder pain. In fact, two mechanical flaws, backward lean in the balance position and early hand separation, correlated with a decreased risk of elbow pain. Two other flaws, a long arm swing and arm ahead of the body at the time of ball release, correlated with a decreased risk of shoulder pain.

\section{DISCUSSION}

Baseball pitching injuries are most commonly due to the accumulation of microtrauma from the repetitive pitching motion. ${ }^{2,15}$ The slow development of these injuries makes it difficult to demonstrate cause and effect. Most serious pitching injuries, those requiring surgery or rehabilitation, occur at the collegiate and professional level. ${ }^{15}$ Many 
TABLE 3

Relationship Between Game Pitch Counts and Joint Pain

\begin{tabular}{|c|c|c|c|c|c|}
\hline \multirow{2}{*}{ Game pitch counts } & \multirow{2}{*}{$\begin{array}{c}\text { No. of } \\
\text { appearances }\end{array}$} & \multicolumn{2}{|c|}{ Elbow } & \multicolumn{2}{|c|}{ Shoulder } \\
\hline & & Odds ratio & $P$ value & Odds ratio & $P$ value \\
\hline \multicolumn{6}{|l|}{ Overall } \\
\hline $1-24$ & 1023 & Referent & 0.07 & Referent & $<0.01$ \\
\hline $25-49$ & 1060 & 1.03 & & 1.15 & \\
\hline $50-74$ & 992 & 1.21 & & 1.23 & \\
\hline $75-99$ & 476 & 1.35 & & 1.52 & \\
\hline $100+$ & 238 & 1.44 & & 1.77 & \\
\hline \multicolumn{6}{|l|}{9 to 10 years old } \\
\hline $1-24$ & 430 & Referent & 0.67 & Referent & 0.01 \\
\hline $25-49$ & 373 & 1.20 & & 0.97 & \\
\hline $50-74$ & 346 & 1.40 & & 1.11 & \\
\hline $75-99$ & 149 & 0.91 & & 1.32 & \\
\hline $100+$ & 90 & 1.33 & & 2.01 & \\
\hline \multicolumn{6}{|l|}{11 to 12 years old } \\
\hline $1-24$ & 319 & Referent & 0.60 & Referent & 0.94 \\
\hline $25-49$ & 353 & 0.84 & & 0.98 & \\
\hline $50-74$ & 319 & 0.83 & & 1.10 & \\
\hline $75-99$ & 164 & 1.30 & & 1.14 & \\
\hline $100+$ & 70 & 0.87 & & 0.76 & \\
\hline \multicolumn{6}{|l|}{13 to 14 years old } \\
\hline $1-24$ & 274 & Referent & 0.06 & Referent & 0.02 \\
\hline $25-49$ & 334 & 1.12 & & 1.51 & \\
\hline $50-74$ & 327 & 1.38 & & 1.65 & \\
\hline $75-99$ & 163 & 1.59 & & 2.17 & \\
\hline $100+$ & 78 & 2.22 & & 2.15 & \\
\hline
\end{tabular}

TABLE 4

Relationship Between Cumulative Season Pitches after an Appearance and Risk of Joint Pain from the Appearance

\begin{tabular}{|c|c|c|c|c|c|}
\hline \multirow{2}{*}{ Season pitches to date } & \multirow{2}{*}{$\begin{array}{l}\text { No. of such } \\
\text { appearances }\end{array}$} & \multicolumn{2}{|c|}{ Elbow } & \multicolumn{2}{|c|}{ Shoulder } \\
\hline & & Odds ratio & $P$ value & Odds ratio & $P$ value \\
\hline $1-200$ & 2126 & Referent & $<0.01$ & Referent & $<0.01$ \\
\hline $201-400$ & 957 & 1.63 & & 1.65 & \\
\hline $401-600$ & 460 & 2.81 & & 2.34 & \\
\hline $601-800$ & 194 & 3.34 & & 2.90 & \\
\hline $800+$ & 52 & 2.61 & & 3.29 & \\
\hline
\end{tabular}

experienced sports medicine professionals, including the senior author (JRA), believe that shoulder or elbow pain in the young pitcher is a warning sign of the development of an overuse injury. Although muscle soreness is normal and necessary in the development of a pitcher, joint pain is not. Thus, shoulder and elbow pain were designated as the outcomes of interest in this study.

The main contributors to shoulder and elbow pain in youth baseball pitchers are believed to be pitch types, pitch counts, and pitching mechanics. In the present study, all three variables were investigated and two were identified as risk factors. Namely, breaking pitches and high pitch counts were demonstrated to produce a significantly increased risk of elbow and shoulder pain among youth baseball pitchers from the ages of 9 to 14 .

The curveball and slider were both found to be related to joint pain in young pitchers. Like the fastball, these pitches place high loads on the shoulder and elbow. ${ }^{8}$ Additionally, the curveball requires the skill of mastering a new set of mechanics. ${ }^{4,9}$ Adult pitchers throw the slider and fastball using similar mechanics ${ }^{4,9}$; however, many expert coaches, including an author of this study (EDO), question whether young pitchers who claim to use a slider are actually throwing a curveball instead. The danger of the loads created by these breaking pitches is magnified for the prepubescent athlete because the growth plates in the elbow and shoulder joints are still open and are therefore more susceptible to stress-related injuries. ${ }^{5,12}$

The change-up in its variety of forms has been demonstrated to be a safe pitch for 9- to 14-year-old baseball pitchers and is recommended for this age group instead of the curveball and slider. Not only is the change-up a safe alternative, it can be just as effective as other breaking pitches because the key to its success is an off-speed that upsets the batter's timing. Youth baseball coaches need to be aware that a change-up can function as the off-speed pitch without the increased risk of elbow or shoulder pain. These pitch type recommendations are consistent with the results of a survey conducted by USA Baseball ${ }^{3}$ that concluded pitchers should first learn the fastball (recommended age, $8 \pm 2$ years $)$, then the change-up $(10 \pm 3$ years), followed by the curveball (14 \pm 2 years), and the slider (16 \pm 2 years).

High pitch counts are associated with arm problems at every level of competition, including youth baseball. These 9- to 14-year-old pitchers were sometimes subjected to 100 
or more pitch appearances while remaining within the league innings-pitched limits. These pitch counts may put the pitchers at risk not only for immediate problems, but also for overuse injury if they continue to play baseball beyond their youth years. Pitchers in this study demonstrated an increased risk of elbow and shoulder pain with an increased number of pitches per game and with an increased number of pitches over the course of the season. This is the strongest evidence to date that pitch limits rather than inning limits might improve the safety of youth baseball. The steady increase in the odds ratio for both shoulder and elbow pain suggests that there is not an obvious optimal pitch limit. Determining how much increased risk is acceptable is a subjective decision; however, it is worth noting that there was a $35 \%$ increased risk of elbow pain and a $52 \%$ increased risk of shoulder pain by the 75-to-99 pitches per game level. The previous USA Baseball survey recommended limits of $52 \pm 15$ pitches per game for 8- to 10-year-old pitchers, $68 \pm 18$ for 11- to 12-year-old pitchers, and $76 \pm 16$ for 13 - to 14 -yearold pitchers. ${ }^{3}$

It is possible that a pitch count limit could be difficult to enforce and might result in coaching behavior that is contradictory to the spirit of the game (for instance, having hitters intentionally take pitches to increase the opposing pitcher's pitch count). A possible solution might be to limit the number of batters faced. Pitchers in the current study averaged approximately five pitches per batter. Therefore, the number of pitches thrown could be moderated by limiting the number of batters a pitcher could face to one-fifth of the ideal pitch count limit. For example, to regulate pitchers to approximately 75 pitches per game, a limit of 15 batters faced could be imposed.

The current study has demonstrated that the number of game pitches thrown during a season is a significant risk factor for elbow and shoulder pain. For elbow pain there was a steadily increasing risk up to the 601-to-800 pitch level. For shoulder pain, this steadily increasing risk went beyond the 800-pitch level. This suggests that the number of cumulative pitches should be limited. The current study did not address the issue of all-star and tournament play. An extended all-star or tournament schedule might result in hundreds of additional pitches on the young arm.

Although the current study showed that too much competitive pitching increases the risk of joint pain, it is important to understand that infrequent competitive pitching can be detrimental to a pitcher's development. Pitching, throwing, and practice drills are vital for a young pitcher to develop the coordination, strength, flexibility, endurance, mentality, and skills necessary for success in the present and future. For example, Crockett et al. ${ }^{6}$ demonstrated that the extraordinary range of shoulder external rotation present in successful professional pitchers is due to adaptations in the growth of the humerus from repetitive loading during pitching in childhood. A pitcher's safe and successful development requires the athlete to maintain a fine line between necessary and appropriate use and overuse.

Pitching mechanics are the third key to safe and effective pitching. Fleisig et al. ${ }^{10}$ showed that similar body position and movement timing patterns are used by successful pitchers, regardless of age and performance level. In the current study we were unable to demonstrate a relationship between improper pitching mechanics and elbow and shoulder pain in young pitchers. Limitations with the mechanical analysis were sample size, data collection methods, and the subjectivity of the model itself. Videotaping was often conducted during preseason evening practices, which required that the camera shutter speeds be reduced for the low light levels. The slower shutter speed made accurate analysis of the video frames difficult. Although the video analysis model represents a good first effort at developing a field tool for evaluating pitching mechanics, revising the model and procedures may improve the ability to achieve consistent results. Although no meaningful associations were found, it is still believed that teaching proper mechanics at a young age will reduce injury risk and increase performance.

This study represents the second game-by-game investigation of arm problems in youth baseball. However, much more can be done to understand pitching injuries and their causes. The authors recommend that a longitudinal study be initiated to follow a cohort of baseball pitchers from their 1st year participating through the end of their pitching career. Such a study could demonstrate the relationship between childhood pitching overuse and subsequent teen or adult arm injury.

It is hoped that the results of this study will assist youth baseball leagues in protecting their young pitchers from harm so that children can enjoy the game without injury and improve their opportunity for success at higher performance levels.

\section{Recommendations}

On the basis of our results, to minimize complaints of shoulder and elbow pain, we recommend that pitchers between 9 and 14 years old do not throw the curveball or slider. These pitchers should use the fastball and change-up exclusively. Baseball organizations may consider limiting pitchers in this age group to 75 pitches in a game and 600 pitches in a season. Alternatively, the number of batters faced during a game and season could be limited to 15 and 120, respectively. Furthermore, pitchers should not be allowed to circumvent pitch limits by participating in more than one league at a time. Full effort pitching should be limited, and all organized throwing sessions should be monitored closely by a coach or parent. The recommended limits refer to full effort, competitive game pitches and do not include warm-up pitches, practice pitches, throwing from other positions, and throwing drills, all of which are vital for a pitcher's development.

\section{ACKNOWLEDGMENTS}

This study was funded by a grant from the USA Baseball Medical \& Safety Committee and a supplemental grant from the U.S. Olympic Committee (SST98-BAS-201). The authors thank Rochelle Nicholls, MS, for her help in data collection and analysis; LeaVonne Pulley, $\mathrm{PhD}$, for her 
help in development of the questionnaires; John Waterbor, $\mathrm{MD}, \mathrm{DrPH}$, for his thoughtful editorial suggestions; and all of the youth players, coaches, and league officials for their participation in the data collection.

\section{REFERENCES}

1. Adams JE: Injury to the throwing arm: A study of traumatic changes in the elbow joints of boy baseball players. Calif Med 102: 127-132, 1965

2. Andrews JR, Fleisig GS: Preventing throwing injuries. J Orthop Sports Phys Ther 27: 187-188, 1998

3. Andrews JR, Fleisig GS: How many pitches should I allow my child to throw? USA Baseball News (April): 5, 1996

4. Barrentine SW, Matsuo T, Escamilla RF, et al: Kinematic analysis of the wrist and forearm during baseball pitching. J Appl Biomech 14: 24-39, 1998

5. Carson WG Jr, Gasser SI: Little Leaguer's shoulder: A report of 23 cases. Am J Sports Med 26: 575-580, 1998

6. Crockett HC, Gross LB, Wilk KE, et al: Osseous adaptation and range of motion at the glenohumeral joint in professional baseball pitchers. Am J Sports Med 30: 20-26, 2002

7. Dillman CJ, Fleisig GS, Andrews JR: Biomechanics of pitching with emphasis upon shoulder kinematics. J Orthop Sports Phys Ther 18: 402408, 1993
8. Escamilla RF, Fleisig GS, Alexander E, et al: A kinematic and kinetic comparison while throwing different types of baseball pitches. Med Sci Sports Exerc 26: S175, 1994

9. Escamilla RF, Fleisig GS, Barrentine SW, et al: Kinematic comparisons of throwing different types of baseball pitches. J App/ Biomech 14:1-23, 1998

10. Fleisig GS, Barrentine SW, Zheng N, et al: Kinematic and kinetic comparison of baseball pitching among various levels of development. J Biomech 32: $1371-1375,1999$

11. Gugenheim JJ, Stanley RF, Woods GW, et al: Little League survey: The Houston study. Am J Sports Med 4: 189-200, 1976

12. Kocher MS, Waters PM, Micheli LJ: Upper extremity injuries in the paediatric athlete. Sports Med 30: 117-135, 2000

13. Larson RL, Singer KM, Bergstrom R, et al: Little League survey: The Eugene study. Am J Sports Med 4: 201-209, 1976

14. Lyman S, Fleisig GS, Waterbor JW, et al: Longitudinal study of elbow and shoulder pain in youth baseball pitchers. Med Sci Sports Exerc 33: 18031810, 2001

15. Oberlander MA, Chisar MA, Campbell B: Epidemiology of shoulder injuries in throwing and overhead athletes. Sports Med Arthrosc Rev 8: 115-123, 2000

16. Pasternack JS, Veenema KR, Callahan CM: Baseball injuries: A Little League survey. Pediatrics 98: 445-448, 1996

17. Torg JS, Pollack H, Sweterlitsch P: The effect of competitive pitching on the shoulders and elbows of preadolescent baseball players. Pediatrics 49: 267-272, 1972 